\title{
Microsurgical Resection of Petroclival Tumors via the Subtemporal Transtentorial Approach
}

\author{
Zhou XU', Xingruo ZENG ${ }^{2}$, Daofeng TIAN ${ }^{1}$, Qianxue CHEN ${ }^{1}$ \\ ${ }^{1}$ Renmin Hospital of Wuhan University, Department of Neurosurgery, Wuhan, China \\ ${ }^{2}$ The Central Hospital of Wuhan, Wuhan, China
}

\section{ABSTRACT}

AIM: To study and summarize our experience with the subtemporal transtentorial approach for resection of petroclival tumors.

MATERIAL and METHODS: Twenty-eight patients who suffered from petroclival tumors and underwent tumor resection via a subtemporal transtentorial approach between October 2010 and July 2013 were retrospectively reviewed.

RESULTS: Total resection was performed in 22 cases, at a rate of $78.57 \%$, subtotal resection in 5 cases, and large portion resection in 1 case. 13 patients who suffered from varying degrees of postoperative nerve dysfunction all improved or recovered, during the 6 to 24 month post-operative follow up.

CONCLUSION: The subtemporal transtentorial approach is one of the best approaches for resection of petroclival tumors, especially for a tumor at the petrosal apex and upper-middle clival region, and has the advantages of less trauma, easy procedure of craniotomy, and also a good surgical exposure.

KEYWORDS: Microsurgery, Subtemporal transtentorial approach, Petroclival tumor

\section{INTRODUCTION}

Petroclival tumor, a kind of deep-seated lesion of the skull base, always involves the petrosal bone and clivus at the same time, and it can also invade the cavernous sinus and the sellar region, and often encases multiple cranial nerves and the blood vessels of the skull base. However, it is always hard to ensure good surgical exposure during operation and the tumor is difficult to resect completely. Sometimes even severe postoperative complications may occur. It is therefore one of the most arduous challenges in neurosurgery. From October 2010 to July 2013, 28 cases of petroclival tumor were resected via the subtemporal transtentorial approach in our department. The intraoperative and postoperative complications and surgical outcomes of these patients were collected and reviewed.

\section{MATERIAL and METHODS}

\section{Patient Population}

In this study, 28 patients who suffered from petroclival tumors were treated surgically from October 2010 to July 2013. The study group consisted of twelve males and sixteen females. The mean age was 45.2 years and ranged from 24 to 67 years. There were 20 cases with meningioma, 4 cases with trigeminal neurinoma, 3 cases with chordoma, and 1 case with cholesteatoma.

\section{Clinical Presentation}

The most frequent clinical manifestations were headache and dizziness in 25 cases, facial numbness in 8 cases, diplopia in 6 cases, dysecoia in 4 cases, myodynamia decrudescence in 8 cases, and coordination disturbance in 4 cases. The mean duration of symptoms was 32 months, ranging from 2 to 84 months. 


\section{Imaging}

The preoperative imaging examination must include contrastenhanced magnetic resonance imaging (MRI) in order to understand the tumor's location, extension direction, and evaluate the relationship between the tumor and surrounding important structures. CT angiography was also performed in some cases to evaluate the blood supply of the tumors. The clivus was divided into the upper clivus, middle clivus and lower clivus by two anatomical markers, Dorello's canal and the nerve part of the jugular foramen (6). The tumor was located at the upper clivus and petrosal apex in 11 cases, upper-middle clivus in 17 cases, there was extension to the parasellar and cavernous sinus in 15 cases, and brainstem compression was observed in all 28 cases. The longest diameter of the tumors was between $2.5 \mathrm{~cm}$ to $5.6 \mathrm{~cm}$, and the average $3.8 \mathrm{~cm}$.

\section{Surgical Technique}

The patients were placed supine, with a pad under the ipsilateral shoulder. The head was rotated to the contralateral side and secured in a Sugita head holder, keeping the zygomatic arch horizontal to the floor. A subtemporal transtentorial approach was used through temporal flap craniotomy, and the inferior margin of the bone window should be down as far as possible to the bottom of the middle cranial fossa. If the mastoid air cell is opened during the craniotomy, it should be sealed completely by bone wax. The temporal lobe should be carefully retracted to expose the free margin of the tentorium, and we especially need to pay attention to protect the vein of Labbé. If necessary, the temporal pole and inferior temporal gyrus can be resected for better surgical exposure. It is important to incise the perimesencephalic cistern to evacuate the cerebrospinal fluid (CSF) for further exposure. In order to acquire a better exposure of the subtemporal structures, the tentorium was incised parallel to the posterior part of superior petrosal sinus to the free margin of the cerebellum tentorium, and one should be careful to protect the trochlear nerve. After exposure, exploring and coagulating the basal parts of the tumor may contribute to the reduction of the blood supply of the tumor, and then tumor debulking was performed till resected totally, and at the same time carefully identify the relationship between the tumor and the important structures such as the brainstem, vessels, cranial nerve and petrosum. If necessary, the petrosal apex bone and the bone crest of the superior and posterior parts of the internal acoustic meatus can be removed with a high-speed grinding drill to expose the petrosal dorsum, middle-lower clivus and the structures around the internal acoustic meatus.

\section{Criterion of Tumor Resection and Therapeutic Evaluation}

The tumor resection criterion was as Yang et al. (8) described according to the intraoperative view and postoperative MRI findings. Total resection: no tumor residual; subtotal resection: tumor residual less than 10\%; large partial resection: tumor residual less than $50 \%$, but more than $10 \%$. The removed tumor specimens went through pathological diagnosis as routine. Postoperative symptom remission was observed, and we evaluated whether new neurological deficits appeared.

\section{RESULTS}

Among the 28 patients, total resection was performed in 22 cases $(78.57 \%)$, subtotal resection in 5 cases $(17.86 \%)$, and large portion resection in 1 case (3.57\%). In the 20 cases of meningioma, we achieved total resection in 17 cases, subtotal resection in 2 cases, and large portion resection in 1 case. In the 4 cases of trigeminal neurinoma, we achieved total resection in 4 cases; in 3 cases of chordoma, we achieved subtotal resection in the 3 cases; and in 1 case of cholesteatoma we achieved total resection. Compared with preoperative imaging data, the postoperative imaging data were satisfactory (Figure $1 A-D, 2 A-D)$.

The preoperative symptoms, such as headache, dizziness, facial numbness, diplopia, myodynamia decrudescence, coordination disturbance and so on, all were alleviated in varying degrees. 13 patients suffered from new neurological deficit after surgery, oculomotor nerve dysfunction in 5 cases $(17.86 \%)$, trochlear nerve dysfunction in 4 cases (14.29\%), trigeminal nerve symptoms in 6 cases $(21.43 \%)$, abducens nerve dysfunction in 4 cases (14.29\%), facial nerve dysfunction in 4 cases (14.29\%), hemiplegia in 3 cases $(10.71 \%)$, and aphasia in 2 cases $(7.14 \%)$. CSF leakage occurred in 2 cases, and was treated by lumbar cistern drainage. Two patients suffered disturbance of consciousness after surgery, one of which underwent decompression craniectomy due to postoperative brain swelling, and another one underwent intracranial hematoma removal surgery due to the postoperative epidural hematoma.

During the 6-24 months post-operative follow up (the mean follow up period was 16.9 months), the neurological conditions were checked by the same neurosurgeon who performed the operation: total recovery of oculomotor nerve function was observed in 3 cases and partial recovery in 2 cases; total recovery of trochlear nerve function was observed in 4 cases; total recovery of trigeminal nerve function was observed in 4 cases and partial recovery in 2 cases; total recovery of abducens nerve function was observed in 3 cases and partial recovery in 1 case; and improvement of facial nerve function was observed in 4 cases. The myodynamia of the 3 cases with hemiplegia all improved to grade IV-V; 2 cases with aphasia completely recovered within 1 month and 6 months (Table I). Six patients with tumor residual underwent radiotherapy and no recurrence or regrowth were found.

\section{- DISCUSSION}

Over the past decades, resection of petroclival tumor by microsurgery has still been a great challenge to neurosurgeons due to its deep location in the skull base, and the properties of eroding cranial nerves and important vessels, compressing the brain stem, and extending to the cavernous sinus and parasellar region in some of the cases. Several kinds of surgical approaches for resection of petroclival tumors have been developed during the past years, such as transpetrosal presigmoid approach, petrosal approach (divided into retrolabyrinthine, translabyrinthine, transotic, and transcochlear approaches) (2), retrosigmoid approach, subtemporal transtentorial approach, and so on. 

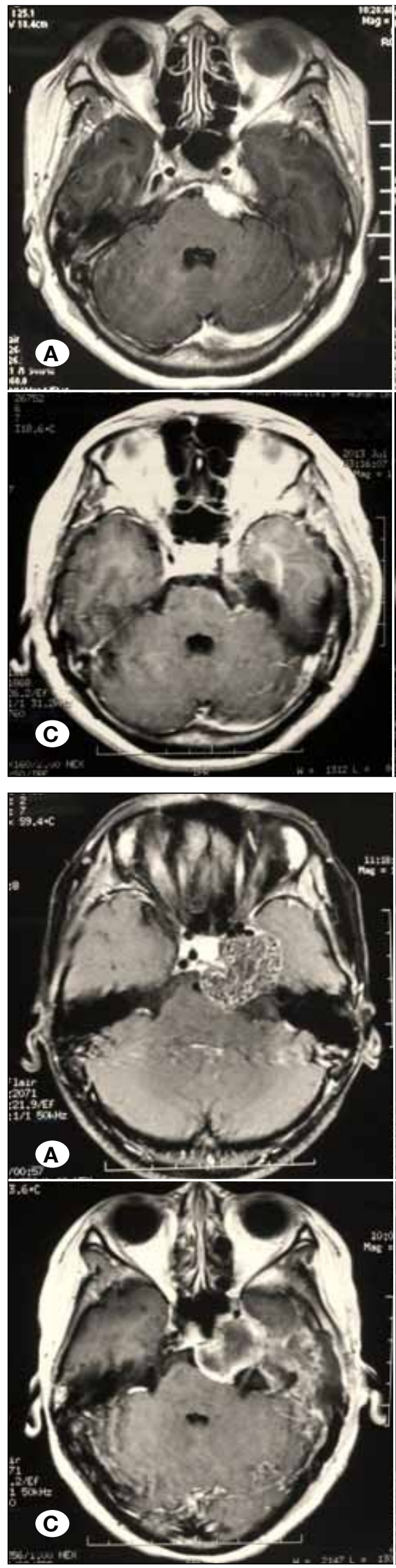
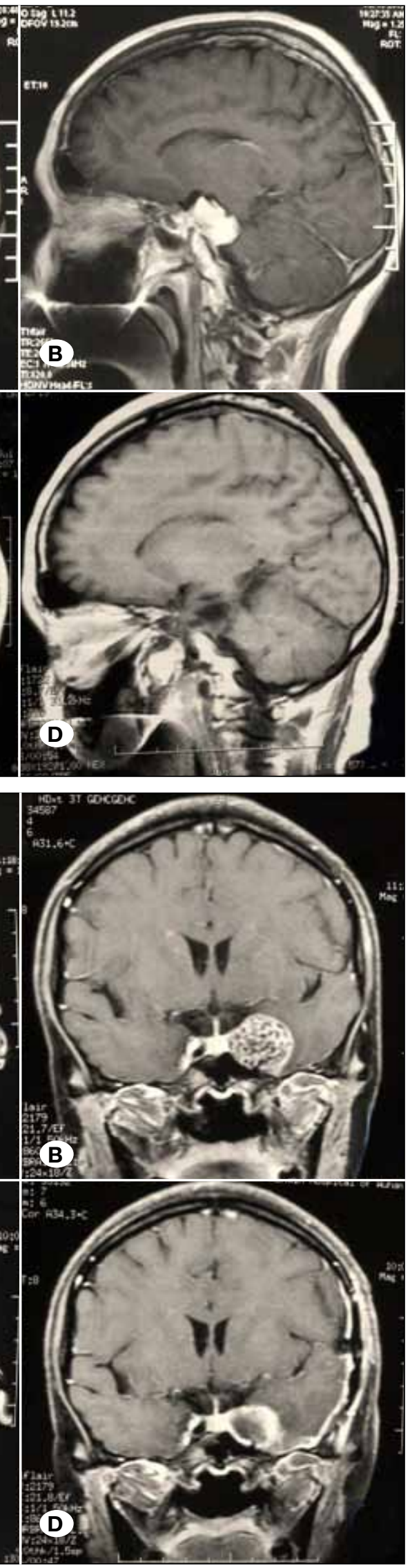

Figure 1: Petroclival meningioma located at the upper clivus and petrosal apex (A,B). We performed total resection (C,D).

Figure 2: Petroclival chordoma extension to the parasellar and cavernous sinuses $(\mathbf{A}, \mathbf{B})$. We performed subtotal resection (C,D). 
Table I: Postoperative Complications and Recovery

\begin{tabular}{lccc}
\hline Postoperative complications & No. of cases & Total recovery cases & Partial recovery cases \\
\hline Nerve III palsy & 5 & 3 & 2 \\
\hline Nerve IV palsy & 4 & 4 & 0 \\
\hline Nerve V palsy & 6 & 3 & 1 \\
\hline Nerve VI palsy & 4 & 0 & 4 \\
\hline Nerve VII palsy & 4 & 1 & 2 \\
\hline Hemiplegia & 3 & 2 & 0 \\
\hline Aphasia & 2 & 2 & 0 \\
\hline CSF leakage & 2 & 1 & 1 \\
\hline Consciousness disturbance & 2 & 4 & 2 \\
\hline
\end{tabular}

In this study, all the 28 cases of petroclival tumor were treated through a subtemporal transtentorial approach. The total resection ratio was $78.57 \%$, and no patients died perioperatively. Our experience is that the subtemporal transtentorial approach should be a preferred approach for the resection of petroclival tumor, especially for a tumor located at the uppermiddle clival region and petrosal apex.

The main body of the tumor is located in the posterior fossa, and a tumor located lower than the internal acoustic meatus may not suitable to this approach $(1,4)$. The procedure of this approach is simpler than the canonical presigmoid approach, and easier to master for the neurosurgeons. It shortens the operation time, and is less affected by anatomic variations of the sigmoid sinus and bulbus venae jugularis (5). Most of the time, only a small part of the petrous bone needs to be removed to acquire good surgical exposure for the basal parts of the tumor, so that the blood supply of the tumor can be cut off at an early stage, and it is important for reducing bleeding and keeping the operation field clear.

When performing this approach, one should consider that excessive retraction of temporal lobe may lead to brain contusion and increase the risk of venous infarction of the vein of Labbé (7), which can cause brain swelling, hemiplegia and aphasia. The use of the gelatin sponge brain retraction technique (3) can greatly reduce the damage, and can provide better surgical exposure as well, due to its ability of absorbing plenty of CSF. When one places the gelatin sponge to the surface and the surface of the temporal lobe, it can absorb the CSF gradually, and accompany with the temporal lobe was retracted mildly along the form of the cortical surface. After exposure of the free margin of the tentorium, in order to avoid of injury of trochlear nerve, we always choose the incision point of the tentorium one centimeter right behind the position that the trochlear nerve enters into the free margin of the cerebellum tentorium, and then a good exposure can be acquired for the upper clivus, anterolateral brainstem, basilar artery and its bifurcation, and also the cranial nerve III and IV. The middle-lower clivus can be seen clearly, and can reach the posterior cranial fossa to deal with the subtentorial parts of the tumor. If the tumor encases the cranial nerves such as the oculomotor nerve, trochlear nerve, and abducens nerve tightly, sharp dissection should be used to detach the tumor tissue from the nerve, and one can even retain a little of the tumor tissue adherent tightly to the nerve, in order to protect the function of the cranial nerve as far as possible. A petroclival tumor always has a close relationship with the brain stem and it often invades the subarachnoid space, destroys the arachnoid and pia mater, results in the confluence of the brainstem vessels and tumor vessels, and causes brainstem edema. If the brain stem was compressed heavily by the tumor, the tumor should be resected step by step under the microscope, and then the brainstem will be dissected from the tumor gradually. If the tumor capsula adheres tightly to the brainstem, it is not safe to perform total resection, so one should leave a little tumor tissue or capsula on it to avoid of postoperative brainstem edema and vasospasm effectively, and obtain a better outcome for the patients. Sometimes the tumor can invade the cavernous sinus and massive haemorrhage may occur during the operation. So one should cut off the main tumor blood supply vessels in advance and then perform tumor resection, and using gelatin sponge or hemostatic gauze to tamp the tumor cavity can reduce bleeding significantly. For a giant tumor, tumor resection can be performed simultaneously to reduce the tumor volume during the processing of basal parts of the tumor, and the surgical exposure will be better.

\section{CONCLUSION}

The choice of surgical approach for petroclival tumors should follow the principle of better surgical exposure, shorter approach, maximum nerve function preservation, and fewer postoperative complications. The subtemporal transtentorial approach has the above advantages for resection of petroclival tumors, especially for a tumor at the petrosal apex and upper-middle clival region. Preoperative imaging studies to understand the tumor's location, extension direction, and the relationship with important structures, can be a great guidance to choose the surgical approach. The right choice of surgical approach and neurosurgeon's skilled microsurgery technique are key to a successful operation. 


\section{REFERENCES}

1. Danner C, Cueva RA: Extended middle fossa approach to the petroclival junction and anterior cerebellopontine angle. Otol Neurotol 25:762-768, 2004

2. Horgan MA, Anderson GJ, Kellogg JX, Schwartz MS, Spektor S, McMenomey SO, Delashaw JB: Classification and quantification of the petrosal approach to the petroclival region. J Neurosurg 93:108-112, 2000

3. Kashimura H, Ogasawara K, Kubo Y, Kakino S, Sasoh M, Takahashi H, Suzuki K, Ogawa A: Brain retraction technique using gelatin sponge in the subtemporal approach. Neurol Med Chir 48:143-146, 2008

4. Little KM, Friedman AH, Sampson JH, Wanibuchi M, Fukushima T: Surgical management of petroclival meningiomas: Defining resection goals based on risk of neurological morbidity and tumor recurrence rates in 137 patients. Neurosurgery 56:546559,2005
5. Seifert V, Raabe A, Zimmermann M: Conservative (labyrinthpreserving) transpetrosal approach to the clivus and petroclival region--indications, complications, results and lessons learned. Acta Neurochir (Wien) 145:631-642, 2003

6. Sekhar LN, Jannetta PJ, Burkhart LE, Janosky JE: Meningiomas involving the clivus: A six-year experience with 41 patients. Neurosurgery 27:764-781, 1990

7. Tahara A, de Santana PA Jr, Calfat Maldaun MV, Panagopoulos AT, da Silva AN, Zicarelli CA, Pires de Aguiar PH: Petroclival meningiomas: Surgical management and common complications. J Clin Neurosci 16:655-659, 2009

8. Yang J, Liu YH, Ma SC, Wei L, Lin RS, Qi JF, Hu YS, Yu CJ: Subtemporal transtentorial petrosal apex approach for giant petroclival meningiomas: Analyzation and evaluation of the clinical application. J Neurol Surg B 73:54-63, 2012 